\title{
Celebrating 50 Years of Experimental Mechanics
}

\author{
Horacio D. Espinosa
}

Published online: 1 December 2009

(C) Society for Experimental Mechanics 2009

This year, Experimental Mechanics celebrates its 50th anniversary. The Journal was launched in January of 1961 by the then Society for Experimental Stress Analysis (SESA) now the Society for Experimental Mechanics (SEM). Benjamin J. Lazan, who is credited as the individual most responsible for the creation of the Journal, wrote an editorial in the first issue of Experimental Mechanics where he articulated the reasons for the creation of the journal. He pointed out that the new journal was the result of the society growth, the broader realm of experimental mechanics, and the role of experimental studies in verification and improvement of theory. In his visionary editorial, Lazan wrote "the solution of the increasingly complex engineering problems must rely more and more on experimental mechanics studies to indicate limitations in current theories, to formulate more realistic and general, yet simple, assumptions required for developing new theories, and later to check the compatibility of these new theories with still newer engineering situations or simulations thereof."

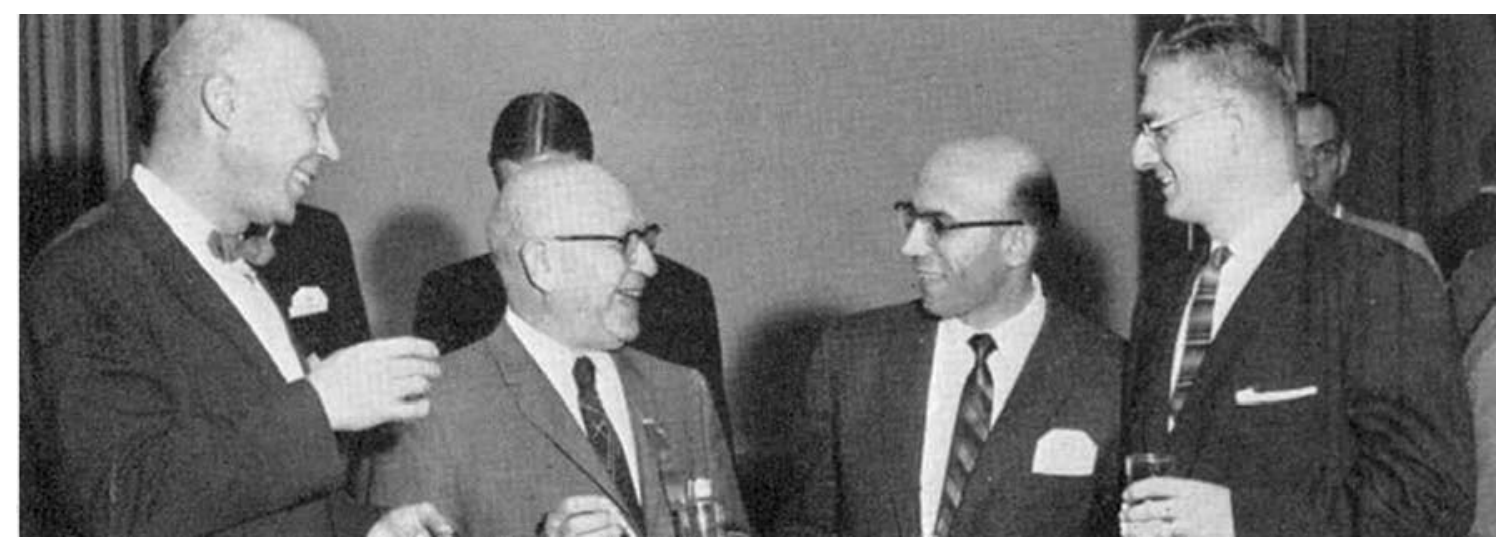

From left: Past-president B.J. Lazan, Honorary Member M.M. Frocht, Executive Secretary Bonney Rossi, and Pastpresident E. Wenk, Jr. This group had much to do with the early success of Experimental Mechanics.

H.D. Espinosa ( $\square$ SEM Fellow)

Department of Mechanical Engineering, Northwestern University, Evanston, IL, USA

e-mail: espinosa@northwestern.edu
It is worth noting that the creation of Experimental Mechanics followed by only a few years the launch of the Russian satellite, Sputnik, which most historians relate to an unprecedented growth of research and education in the USA and other countries. Indeed, developments in space, nuclear and rocketry technologies created the need for the development and characterization of novel materials, structures and systems with unparalleled levels of performance. 
Another interesting historical fact, probably well-known only by those who contributed to the creation of Experimental Mechanics, is that the start-up of the Journal was funded by a grant of $\$ 45,000$ from the National Science Foundation. The specific purpose of the grant was to defray the expenses of organizing and producing the monthly journal for three years, at which time the journal was expected to be self-supporting.

In the early years, Experimental Mechanics served not only as a technical journal, but it also included extensive coverage of Society news and events, and accepted paid advertizing. As the only publication of the Society, it served as the primary communications vehicle with the SESA membership. Experimental Mechanics took the form it has today with the first issue of 1983. At that time, the publication Experimental Techniques was revamped and all of the additional information, including advertizing, that appeared in Experimental Mechanics, other than technical articles, was moved to Experimental Techniques. Experimental Mechanics was then the Society's "archival-quality" journal. In addition to the change in content, in 1983 the frequency of Experimental Mechanics publication was changed from monthly to quarterly. In 2004, in response to a growing backlog, Experimental Mechanics moved from 4 to 6 issues per year.

The journal popularity also grew significantly in recent years, which once again resulted in significant backlog of manuscripts published online. This success prompted the editorial board and the publisher to increase the number of issues from 6 to 9 in 2010. With a 2008 Impact Factor of 1.469 , the highest for the journal since impact factors started to be published by ISI Web of Knowledge, Experimental Mechanics now ranks 3rd overall by Impact Factor, and 2nd by total cites, in the ISI Category of Materials Science, Characterization and Testing. I expect the impact of the Journal to continue to increase in the years to come because Experimental Mechanics is the premier experimental journal in the field of mechanics, which has the unique role of bridging the gap between experimentation and theory. Due to major progress in the development of new experimental tools, with unprecedented temporal and spatial resolution, the experimental community is poised to fulfill such challenge across multiple length (starting at the atomic level) and time scales.

Currently the journal covers a variety of topics including research in solid and fluid mechanics to fields at the intersection of disciplines including physics, chemistry and biology. Development of new devices and technologies for metrology applications in a wide range of industrial sectors (e.g., manufacturing, high-performance materials, aerospace, information technology, medicine, energy and environmental technologies) are also covered. The journal aims to be the voice of the worldwide experimental mechanics community.
Today we have a well established and successful journal. However, now more than ever the role of experimental mechanics is to define the limits and shortcomings of theories as well as to advance discoveries of new phenomena that typically emerge from innovative experimentation. In this regards, the future success of the Journal will continue to depend on the ingenuity of contributing authors, the voluntary service of technical editors, and the commitment of SEM through its members.

In this celebratory year, Experimental Mechanics will publish two special issues and a series of review articles on topics the Journal has covered over the years. The review and timely publications of these articles are being coordinated by Eric Brown, who proposed such initiative to celebrate the 50th anniversary.

Looking at the future, it is likely that national and global initiatives in response to major challenges in energy, health care, and green technologies will shape the content of Experimental Mechanics in coming years. The journal must remain responsive to these global trends and continue to adapt to major waves of technological innovation. In order to continue to play a major role in the dissemination of research and applications of relevant technologies, the journal content must remain contemporary while preserving the Journal tradition of rigorous peer review and publication of in-depth manuscripts.

With the reaching of the 50th anniversary, it is most appropriate to pay tribute to those who contributed to make Experimental Mechanics the outstanding journal it is today. A list of individuals who serve as the Senior Technical Editors since the journal inception is:

\begin{tabular}{lll}
\hline R. Guernsey, Jr & H. Brinson & C.E. Harris \\
C.S. Barton & I.M. Daniel & J.L. Turner \\
A.S. Kobayashi & W.N. Sharpe, Jr. & R. Prabhakaran \\
W.F. Riley & C.P. Burger & A. Shukla \\
R. Mark & D.H. Morris & Y.J. Chao \\
R. Papirno & G.L. Cloud & N.R. Sottos \\
\hline
\end{tabular}

Bonney Rossi was the first Publisher of Experimental Mechanics until he retired in 1978. Since then Tom Proulx continued promoting the journal, as Director of SEM, by working closely with the Editorial Board, the SEM publishing committee, Sage Publications, and more recently Springer. Jennifer Tingets has also been instrumental in the management and growth of the Journal by doing an outstanding job over the years. She accomplished this while working with, and at times guiding, new technical editors.

Horacio D. Espinosa

Senior Technical Editor 